\title{
Interruption Risk Assessment and Transmission of Fresh Cold Chain Network Based on a Fuzzy Bayesian Network
}

\author{
Huanwan Chen $\mathbb{D D}^{1}{ }^{1}$ Qingnian Zhang $\mathbb{D}^{1},{ }^{1}$ Jing Luo, ${ }^{1}$ Xiuxia Zhang, ${ }^{2}$ and Guopeng Chen ${ }^{3}$ \\ ${ }^{1}$ School of Transportation, Wuhan University of Technology, Heping Road No. 1178, Wuchang District, Wuhan City WH 430063, \\ Hubei Province, China \\ ${ }^{2}$ School of Modern Posts, Nanjing University of Posts and Telecommunications, 66 Xinmofan Road, Nanjing City, \\ Jiangsu Province 210003, China \\ ${ }^{3}$ Planning and Operation Department, Hanjiang Water Conservancy \& Hydropower Group Co., Ltd. Hanjiang Building, \\ No. 7 Hangtian Road, Dongxihu District, Wuhan City WH 430048, Hubei Province, China
}

Correspondence should be addressed to Huanwan Chen; chenhw@whut.edu.cn and Qingnian Zhang; zqnwhut@163.com

Received 29 March 2021; Accepted 20 June 2021; Published 7 July 2021

Academic Editor: Luisa Di Paola

Copyright (c) 2021 Huanwan Chen et al. This is an open access article distributed under the Creative Commons Attribution License, which permits unrestricted use, distribution, and reproduction in any medium, provided the original work is properly cited.

\begin{abstract}
The fresh cold chain network is complex, and the interruption risk can significantly impact it. Based on the Bayesian theory, we constructed a fresh cold chain network interruption risk topology structure. The probability of each root node was predicted and calculated based on the fuzzy set theory. The evaluation model was then validated and improved through the virus transmission model based on risk transmission. Sensitivity analysis was used to determine significant risk factors. Several strategies for minimizing interruption risks were identified.
\end{abstract}

\section{Introduction}

In China, the second-largest economy in the world, income levels of domestic residents and consumer demands are increasing. However, integration of the fresh cold chain in China is relatively weak. Furthermore, there is no perfect mechanism for identifying, early warning, and emergency response of interruption risks. COVID-19 has exposed the problem associated with integrating e-commerce platforms and enterprise industrial chains and the management of middle and back-office collaborative construction. Therefore, risk analysis and assessment of the fresh cold chain network interruption should be performed.

Studies have used a fuzzy comprehensive evaluation method to evaluate supply chain risk since its influencing factors are complex [1-4]. However, causal relationship between supply chain factors is unknown. Bayesian networks have also been used to assess supply chain risks, express causal relationships between supply chain factors through directed tree diagrams and conditional probabilities, and calculate their sensitivity [5-9]. A fuzzy best-worst method has been proposed for multiobjective decision making, with regular fuzzy numbers for fuzzy environments [10]. It has been confirmed that two algorithms, the individual decision method $(\mathrm{dm})$, based on multiplicative preference relations, and the GDM method, based on TMPR, can effectively resolve the ambiguity of expert evaluation [11]. Studies have also considered the dynamics between various links of cold chain logistics, as well as successive risk transmission, and used dynamic Bayesian networks for assessment [12, 13]. Guohua [14] evaluated interruption risks of the fresh produce supply chain during the planning horizon, but only for the solutions provided by one-supplier-one-retailer. Yang and Liu [15] evaluated the correlation between supply chain of fresh agricultural products vulnerability and accident risk by constructing a Bayesian model. In Wuhan, an interactive multicriteria group decision making (MCGDM) approach has been proposed for COVID-19 to study effective emergency assistance programs [16]. 
Due to cold chain disruption caused by COVID-19, we postulated that interruption risk depends on various carriers, and spreading, as well as transmission, to other node enterprises and the entire system along the complex network of the fresh cold chain in form of original or new risk is done after the coupling mutation. Risk transmission interruption of fresh cold chain network expands the interruption risk scope, increases the loss or changes, and upgrades nature, causing a substantial effect to cold chain network operation. Therefore, a fresh cold chain network interruption risk assessment model based on Bayesian network theory was constructed to analyze its occurrence probability and major influencing factors in China. The SIR model was included to improve risk transmission. Moreover, interruption risk factors with a significant impact on sensitivity were analyzed, and effective countermeasures were taken to reduce the impact of interruption risk.

\section{Basic Bayesian Network Theory}

JudeA PeArl proposed the Bayesian Network (BAyesiAn Network) in 1988. It is a reasoning network that is based on the uncertainty and variability of probability. The fresh cold chain network covers many companies with mutual synergy and complex structures. Therefore, the impact of the interruption risk source on the network has a high degree of uncertainty. We used Bayesian networks to produce the interruption risk of the fresh and cold chains.

2.1. Bayesian Theorem. If there are variables $X$ and observation data $Y$, the Bayesian network theorem is expressed as

$$
P(X \mid Y)=\frac{P(Y \mid X) P(X)}{P(Y)},
$$

$P(X \mid Y)$ is the posterior probability of variable $X$ under the $Y$ condition, $P(X)$ is the prior probability of $X, P(Y)$ is the probability of $Y$ occurrence, and $P(Y \mid X)$ is the occurrence of variable $X$ under conditional probability of time $Y$ [17].

When $Y$ is a set of observation data, $Y=\left(Y_{1}, \ldots, Y_{m}\right)$, posterior probability of variable $X$ is expressed as

$$
P(X \mid Y)=\frac{P(Y \mid X) P(X)}{P\left(Y_{1}, \ldots, Y_{m}\right)} .
$$

Posterior probability of the Bayesian theorem is updated with the observation data.

When $X$ is a set of data, $X=\left(X_{1}, \ldots, X_{n}\right)$ and $Y$ is a set of observation data, $Y=\left(Y_{1}, \ldots, Y_{m}\right)$, the calculated posterior probability is expressed as

$$
P\left(X_{i} \mid Y_{j}\right)=\frac{P\left(Y_{j} \mid X_{i}\right) P\left(X_{i}\right)}{\sum_{k=1}^{m} P\left(X_{i}\right) P\left(Y_{k} \mid X_{i}\right)},
$$

where $i=1,2, \ldots, n, j=1,2, \ldots, m$.

2.2. Node. The Bayesian network is a directed acyclic graph. Nodes represent random variables, and the node edges represent the causal relationship between variables. In a directed graph, if an edge connects from node $A$ to node $B$, then $A$ is the parent node of $B$, while $B$ is the child node of $A$. $A$ node without a parent is called the root node, and a node without children is called a leaf node.

2.3. Probability Distribution. Each node in the Bayesian network has a probability distribution. Nodes without a parent node (assuming node $A$ ) are assigned a corresponding prior probability distribution $P(A)$, and each child node (taking node $B$ ) is given a conditional probability distribution $P(B \mid \pi(B))$ based on its parent node.

\section{Fresh Cold Chain Network Interruption Risk Assessment Model}

Fresh cold chain network structure analysis revealed that suppliers, manufacturers, distributors, and retailers are mainly used as network nodes. Service providers of various links formed through business interaction and synergy between node enterprises are the network sides. Therefore, the source of supply chain interruption risk is divided into supply chain external risk (external risk), risk between supply chain node enterprises (network collaboration risk), and supply chain node enterprise internal risk sources (internal risk).

\subsection{Topology Structure Construction of the Fresh Cold Chain} Interruption Risk Assessment Model. The fresh cold chain network is complex and interrupted since agricultural production must ensure the quality of fresh products during the production process (preproduction, production, and postproduction) and regional, as well as seasonal, nature of agricultural products. Moreover, the risk source is uncertain. In this section, the Bayesian network was used to construct a topology structure of the fresh cold chain network interruption risk, Figure 1.

The various links and factor settings in the fresh cold chain network interruption risk topology scheme are divided into four categories: the target node, the intermediate node, the subnodes, and the root node, which are the causes of risk release. Table 1 shows node causality of fresh cold chain network interruption risk.

3.2. Node Assignment. The fresh cold chain network is complex and interrupted since agricultural products must ensure the quality of fresh products during the production process (preproduction, production, and postproduction), as well as the regional and seasonal nature of agricultural products. Moreover, the risk source is uncertain. In this section, the Bayesian network was used to construct a topology structure of fresh cold chain network interruption risk.

Bayesian network topology of network interruption risk of the fresh cold chain was constructed based on relevant analysis of network interruption risk source of the fresh cold chain and Bayesian networks. Each Bayesian network node has two states, $Y$ or $N$, where $Y$ indicates the event occurrence and $N$ indicates that the event described did not occur. 


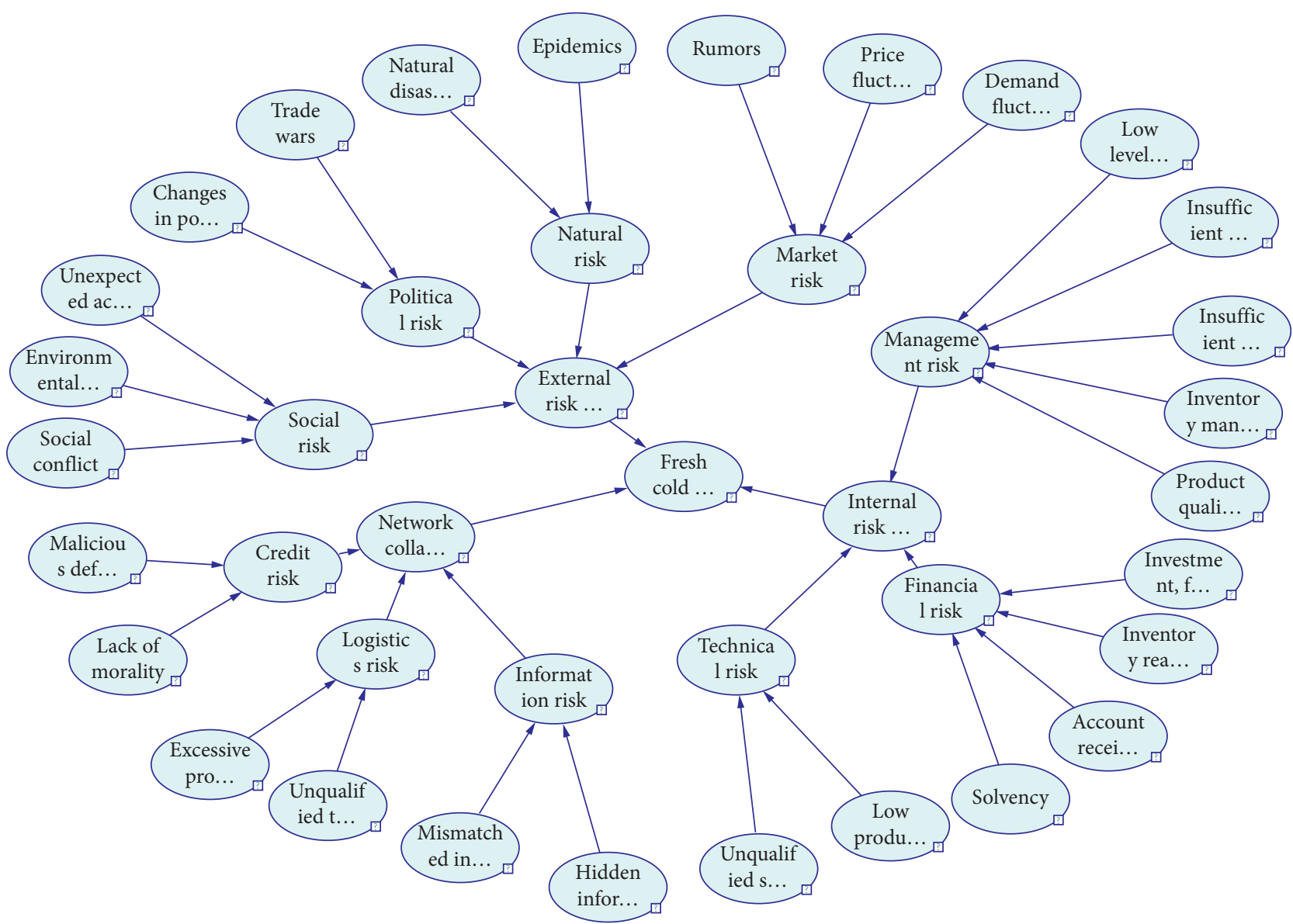

FIGURE 1: Topological structure diagram of the interruption risk of the fresh cold chain.

If a node event occurs ( $Y$ state), its occurrence probability is $P$ and $1-P$ when the node event does not happen ( $N$ state). After establishing the risky Bayesian network structure, it is important to value the nodes (occurrence probability of each node).

Due to a lack of a relatively complete database, expert judgment is used to determine the probability value of risk factors. We used the probability expression proposed by $\mathrm{WiCkens}$ through the seven graded risk occurrence probability fuzzy language variables and their corresponding probability values to unify expert judgment of the probability expression and the possibility of risk occurrence. $\mathrm{WiCkens}$ grouped probability of event occurrence into seven semantic values. The fuzzy number form and $\lambda$ cut set are shown in Table $2[18,19]$.

The following arithmetic average method was used to comprehensively evaluate the results of $n$ experts.

$$
P_{i}=\frac{f_{i 1}+f_{i 2}+f_{i 3}+\cdots+f_{i m}}{n}, \quad(i=1,2, \ldots, m) .
$$

We used the integral value method. Optimization index $\alpha$ reflects the opinions of decision-makers. Assuming that $Q$ is an L-R type fuzzy number, defuzzification value of the fuzzy number $Q$ is obtained;

$$
I(Q)=(1-\mu) I_{R}(Q)+\mu I_{L}(Q)
$$

$\mu \in[0,1]$ is the optimistic coefficient, indicating the upper and lower bounds of the fuzzy number $Q$ defuzzification value $I(Q)$ when $\mu=0$ and $\mu=1$. When $\mu=0.5, I(P)$ is the representative value of the defuzzification value of the fuzzy number $Q$. The integral value of the inverse membership function on the left and right of the fuzzy number is represented by $I_{R}(Q)$ and $I_{L}(Q)$, respectively. For fuzzy triangular numbers, $I_{R}(Q)$ and $I_{L}(Q)$ can also be represented by $\lambda$-cut sets;

$$
\begin{aligned}
& I_{R}(Q)=\frac{1}{2}\left(\sum_{i=0.1}^{1} \lambda_{R}(Q) \Delta \lambda+\sum_{i=0}^{0.9} \lambda_{R}(Q) \Delta \lambda\right), \\
& I_{L}(Q)=\frac{1}{2}\left(\sum_{i=0.1}^{1} \lambda_{L}(Q) \Delta \lambda+\sum_{i=0}^{0.9} \lambda_{L}(Q) \Delta \lambda\right),
\end{aligned}
$$

$\lambda_{R}(Q)$ and $\lambda_{L}(Q)$ are the upper and lower bounds of the $\lambda$-cut set of the fuzzy number $Q(\lambda=0,0.1,0.2, \ldots, 1 ; \Delta \lambda=0.1)$. The Bayesian network is a method used to quantify risk using prior probability distribution as the inference basis. 
TABLE 1: Node causality of fresh cold chain network interruption risk.

\begin{tabular}{|c|c|c|c|}
\hline Target node & Intermediate node & Subnodes & Root node \\
\hline \multirow{20}{*}{$\begin{array}{l}\text { The fresh cold chain network } \\
\text { interruption risk }\end{array}$} & \multirow{10}{*}{ External risk source $(A)$} & \multirow{3}{*}{ Social risk $\left(A_{1}\right)$} & Social conflict $\left(A_{11}\right)$ \\
\hline & & & Environmental pollution $\left(A_{12}\right)$ \\
\hline & & & Unexpected accidents $\left(A_{13}\right)$ \\
\hline & & Political risk $\left(A_{2}\right)$ & Changes in policies and regulations $\left(A_{21}\right)$ \\
\hline & & \multirow{3}{*}{ Natural risk $\left(A_{3}\right)$} & Trade wars $\left(A_{22}\right)$ \\
\hline & & & $\begin{array}{l}\text { Natural disasters }\left(A_{31}\right) \\
\text { Epidemics }\left(A_{32}\right)\end{array}$ \\
\hline & & & Rumors $\left(A_{41}\right)$ \\
\hline & & \multirow[t]{3}{*}{ Market risk $\left(A_{4}\right)$} & Price $\left(A_{42}\right)$ \\
\hline & & & Demand fluctuations $\left(A_{43}\right)$ \\
\hline & & & Low level of information management $\left(B_{11}\right)$ \\
\hline & \multirow{7}{*}{ Internal risk source $(B)$} & \multirow{3}{*}{$\begin{array}{l}\text { Management risk } \\
\qquad\left(B_{1}\right)\end{array}$} & Staff irresponsibility $\left(B_{12}\right)$ \\
\hline & & & Insufficient staff reserves $\left(B_{13}\right)$ \\
\hline & & & $\begin{array}{l}\text { Inventory management risk }\left(B_{14}\right) \\
\text { Product quality and safety }\left(B_{15}\right)\end{array}$ \\
\hline & & \multirow[b]{2}{*}{ Financial risk $\left(B_{2}\right)$} & Investment and financing risk $\left(B_{21}\right)$ \\
\hline & & & $\begin{array}{c}\text { Inventory realization risk }\left(B_{22}\right) \\
\text { Account receivable realization risk }\left(B_{23}\right) \\
\text { Solvency }\left(B_{24}\right)\end{array}$ \\
\hline & & \multirow{2}{*}{ Technical risk $\left(B_{3}\right)$} & Unqualified storage technology $\left(B_{31}\right)$ \\
\hline & & & Low production technology $\left(B_{32}\right)$ \\
\hline & \multirow{3}{*}{$\begin{array}{l}\text { Network collaboration risk } \\
\text { source }(C)\end{array}$} & $\begin{array}{l}\text { Information risk } \\
\qquad\left(C_{1}\right)\end{array}$ & $\begin{array}{c}\text { Mismatched information }\left(C_{11}\right) \\
\text { Hidden information storage or transmission } \\
\text { dangers }\left(C_{12}\right)\end{array}$ \\
\hline & & Logistics risk $\left(C_{2}\right)$ & $\begin{array}{c}\text { Unqualified transportation }\left(C_{21}\right) \\
\text { Excessive product retention time }\left(C_{22}\right)\end{array}$ \\
\hline & & Credit risk $\left(C_{3}\right)$ & $\begin{array}{l}\text { Immorality }\left(C_{31}\right) \\
\text { Malicious default on finances }\left(C_{32}\right)\end{array}$ \\
\hline
\end{tabular}

TABle 2: Fuzzy number form and $\lambda$ cut set.

\begin{tabular}{lcr}
\hline Language description & Fuzzy number form & $\lambda$ cut set \\
\hline Very high $(\mathrm{VH})$ & $f_{\mathrm{VL}}=(0.0,0.1,0.2)$ & $f_{\mathrm{VL}}^{\lambda}=[0.1 \lambda+0,-0.1 \lambda+0.2]$ \\
High $(H)$ & $f_{L}=(0.1,0.2,0.3)$ & $f_{L}^{\lambda}=[0.1 \lambda+0.1,-0.1 \lambda+0.3]$ \\
Faint high $(\mathrm{FH})$ & $f_{\mathrm{FL}}=(0.2,0.3,0.4,0.5)$ & $f_{\mathrm{FL}}^{\lambda}=[0.1 \lambda+0.2,-0.1 \lambda+0.5]$ \\
Medium $(M)$ & $f_{M}=(0.4,0.5,0.6)$ & $f_{M}^{\lambda}=[0.1 \lambda+0.4,-0.1 \lambda+0.6]$ \\
Faint low (FL) & $f_{\mathrm{FH}}=(0.5,0.6,0.7,0.8)$ & $f_{\mathrm{FH}}^{\lambda}=[0.1 \lambda+0.5,-0.1 \lambda+0.8]$ \\
Low $(L)$ & $f_{H}=(0.7,0.8,0.9)$ & $f_{H}^{\lambda}=[0.1 \lambda+0.5,-0.1 \lambda+0.9]$ \\
Very low $(\mathrm{VL})$ & $f_{\mathrm{VH}}=(0.8,0.9,1.0)$ & $f_{\mathrm{VH}}^{\lambda}=[0.1 \lambda+0.8,-0.1 \lambda+1.0]$ \\
\hline
\end{tabular}

$$
\begin{aligned}
& P\left(A_{1}=Y\right)=\sum P\left(A_{11} ; A_{12} ; A_{13} ; A_{1}=Y\right)=P\left(A_{11}=Y ; A_{12}=Y ; A_{13}=Y ; A_{1}=Y\right)+P\left(A_{11}=N ; A_{12}\right. \\
& \left.\quad=Y ; A_{13}=Y ; A_{1}=Y\right)+\cdots+P\left(A_{11}=N ; A_{12}=N ; A_{13}=N ; A_{1}=Y\right) .
\end{aligned}
$$

The occurrence probability of node "social risk" was calculated using equation (5). Similarly, occurrence probabilities of nodes $A, B, C, A_{2}, A_{3}, A_{4}, B_{1}, B_{2}, B_{3}, C_{1}, C_{2}$, and $C_{3}$ were calculated.

In the constructed Bayesian network, it was assumed that node external risk $A$, internal risk $B$, and network collaboration risk $C$ are independent:

$$
\begin{aligned}
& P(T=Y ; A=Y ; B=Y ; C=Y)=P(A \\
& \quad=Y) P(B=Y) P(C=Y) P(T=Y \mid A=Y ; B=Y ; C=Y) .
\end{aligned}
$$

Equations (7) and (8) were used to calculate prior and conditional probabilities of each node. The Bayesian network structure was first established, the relationship between the nodes explained, and conditional probability table formulated to calculate the posterior probability of a single node (reasoning and solving the probability of fresh cold chain interruption).

\section{Risk Assessment Model of Fresh Cold Chain}

4.1. Priori Model for Fresh Cold Chain Interruption Risk Assessment. In the previous section, we introduced the process of constructing the Bayesian network fresh cold chain network interruption risk assessment model and its importance in evaluating the interruption risk status. Occurrence probability of the network interruption risk of 
the fresh cold chain network was obtained based on experts' prior and conditional probability, as shown in Figure 2.

Values corresponding to the seven set fuzzy languages were as follows: very high $(\mathrm{VH})=0.925$, high $(H)=0.825$, faint high $(\mathrm{FH})=0.7$, medium $(M)=0.525$, faint low $(\mathrm{FL})=0.4$, low $(L)=0.225$ and very low $(\mathrm{VL})=0.125$. It was found that the network interruption risk in China's fresh cold chain network was 0.38 . Its occurrence probability was between faint low $(\mathrm{FL})=0.4$ and low $(L)=$ 0.225 , but closer to the faint low state. The fresh cold chain network in China is relatively stable. The external risk significantly impacts the interruption risk of the fresh cold chain network. However, political and natural risks substantially influence the external risk. Based on the current situation, the major sources of interruption risk are trade wars and epidemics.

The probability of a node interrupting the risk source is only the occurrence probability, while the probability of conditional influence on child nodes after its occurrence and external risk occurrence is often followed by government control measures and various social assistance strategies, while reducing, to some extent, the influence degree of external risks. For instance, during COVID-19 in 2020, Wuhan City was closed, traffic was interrupted, people were isolated at home, and their life pace was interrupted. However, the whole country was united to fight the pandemic, and various social assistance measures were put in place, thereby easing social life. It is necessary to improve influencing variables of external risks for model accuracy.

China is a humanitarian and people-oriented country (putting people first). During natural disasters, China urgently provides emergency rescue and relief to minimize death. During social conflicts, China dispatches its armed forces for a quick resolution, minimizing the impact of the conflict. Social assistance reduces the impact of social and natural risks to a certain extent. Therefore, the node "social assistance" can be used as the parent node of "social risk" and "natural risk." The first step of node "social assistance" was calculated using equation (5) (the probability of the test and conditional probability of modifying the two nodes of "social risk" and "natural risk").

The government regulates abnormal price fluctuations in the market through macrocontrol measures. It also clarifies information through communication platforms, such as the Internet, and enforces coercive measures to reduce the damage caused by misinformation. Therefore, the node "government control" is the parent node of the two nodes "price fluctuation" and "misinformation," used to calculate prior probability of the node "government control" according to "Node assignment" and modify the two nodes (Conditional probability of the node).

Evaluation results (Figure 3) show that the interruption probability of the fresh cold chain network was reduced. Analysis of the revised evaluation results indicates that the probability of the fresh cold chain network interruption risk was 0.3 , which is between faint low $(\mathrm{FL})=0.4$ and low $(L)=$ 0.225 , but closer to the low state.
Internal risk exerts the most significant impact on fresh cold chain network interruption risk. However, management and technical risks are major internal risk effects. Currently, the low level of information management by employees, irresponsibility, insufficient personnel reserves, low production technologies, and unqualified storage technology are the major sources of interruption risk.

\subsection{SIR Model Simulation Interrupts Risk Propagation.}

Construction of an SIR model is the propagation process used to simulate risk in the fresh cold chain network to better understand the degree of influence on the network after its interruption risk for graduate students. In the fresh cold chain network, the risk in node companies can be passed to continuous node companies. There are three node companies in the system: node company $S$ that is susceptible to infection risk, node company $I$ that is the susceptible, and risk Immune Node Enterprise $R$. The probability that a type $S$ node company is infected by a type I node company is the probability that a type $I$ node company obtains immunity after the risk elimination. Regarding the time $S, I$, and density of the node enterprise in the $R$ state, the dynamic behavior of risk propagation in the SIR model is calculated as follows:

$$
\left\{\begin{array}{l}
\frac{\mathrm{d} s(t)}{\mathrm{d} t}=-\alpha i(t) s(t), \\
\frac{\mathrm{d} i(t)}{\mathrm{d} t}=\alpha i(t) s(t)-\beta i(t), \\
\frac{\mathrm{d} r(t)}{\mathrm{d} t}=\beta i(t) .
\end{array}\right.
$$

The fuzzy Bayesian network fresh cold chain network risk assessment shows that if there are no government control measures and various social assistance strategies, risk occurrence probability is 0.38 . However, risk occurrence probability is $0.3,0.38$, and 0.3 with government control measures and various social assistance strategies, respectively. Government control measures and various social assistance strategies reduce infection risk probability and increase the probability of node enterprises gaining risk immunity after risk elimination. We used a small network of fresh cold chains with 1000 nodes, four domain nodes, and a reconnection probability of 0.2 to determine whether to adopt government control measures and assess risk propagation for various social assistance strategies Figures 4 and 5.

In the presence of government control measures and various social assistance strategies, the infection risk peak of enterprises reduced from 652 to 373 , the infection scale decreased by $40.22 \%$, and risk peak of infection retreated from 8 to 12 , while the duration of risk transmission decreased from 69 to 45. Analysis of transmission results of the interruption risk model revealed that the impact of interruption risk on the cold 


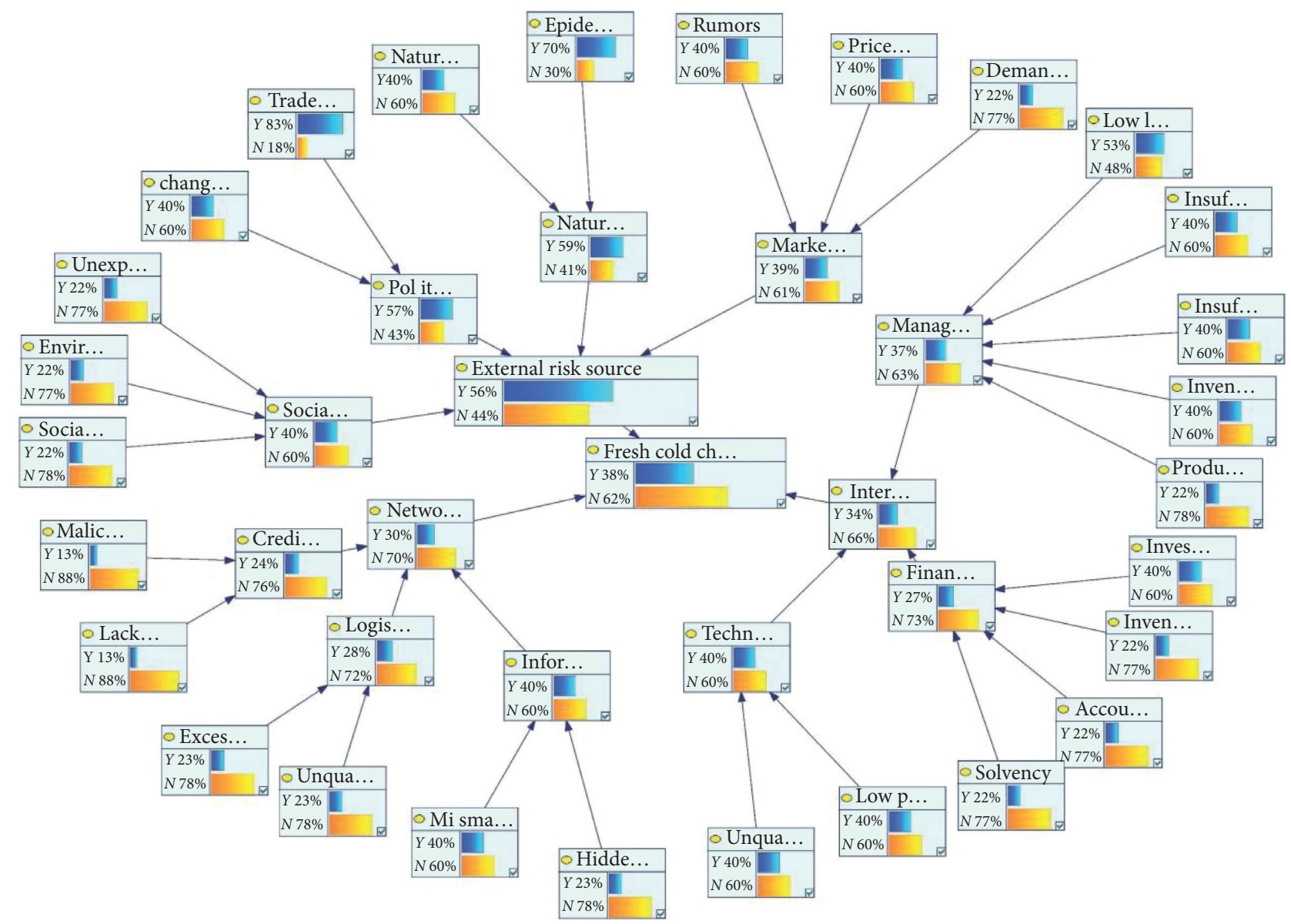

FIGURE 2: Risk assessment of fresh cold chain network interruption based on prior probability.

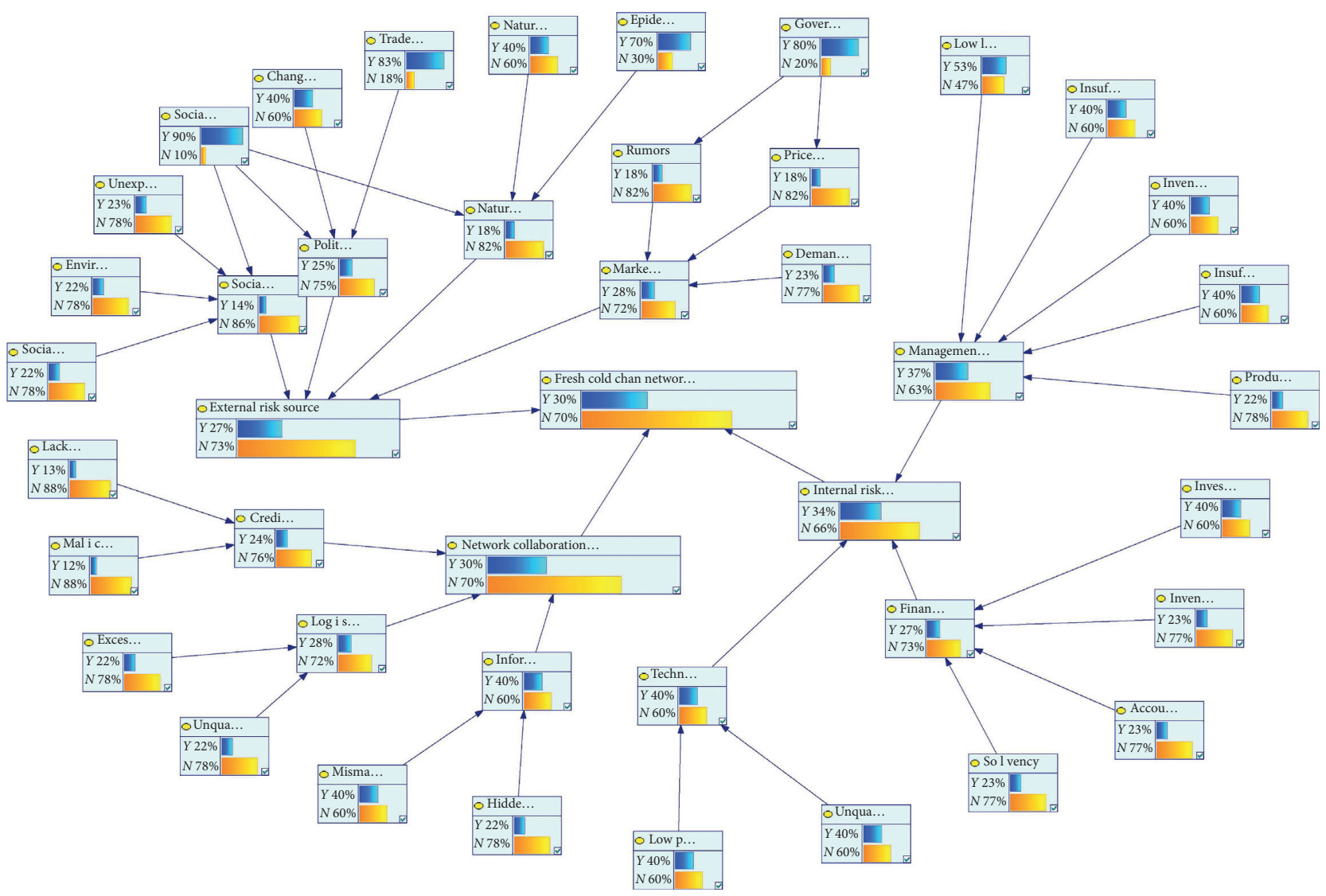

FIgURE 3: Revised fresh cold chain network interruption risk assessment. 


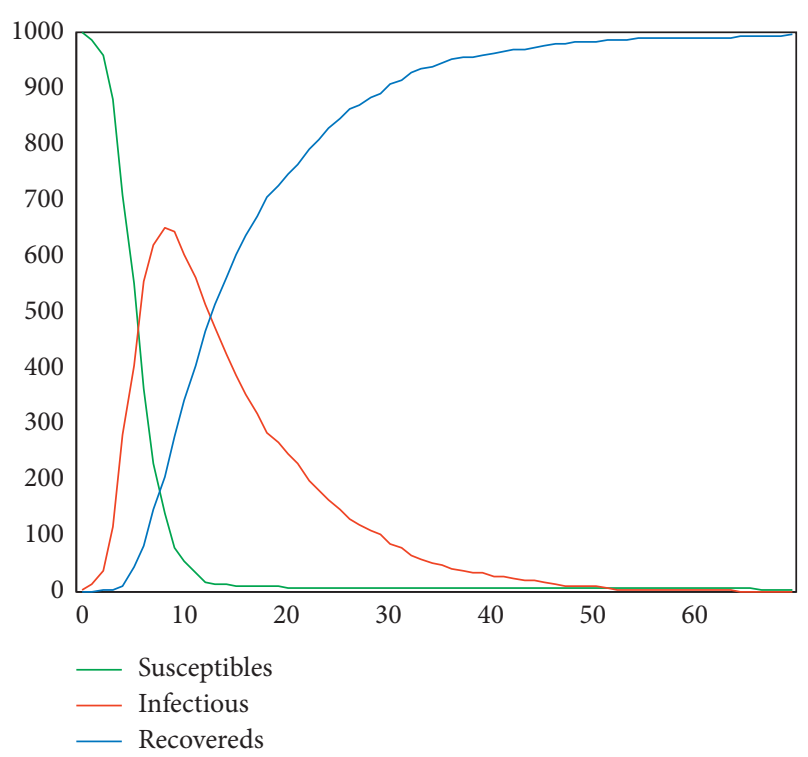

FIGURE 4: No government control measures and various social assistance strategies.

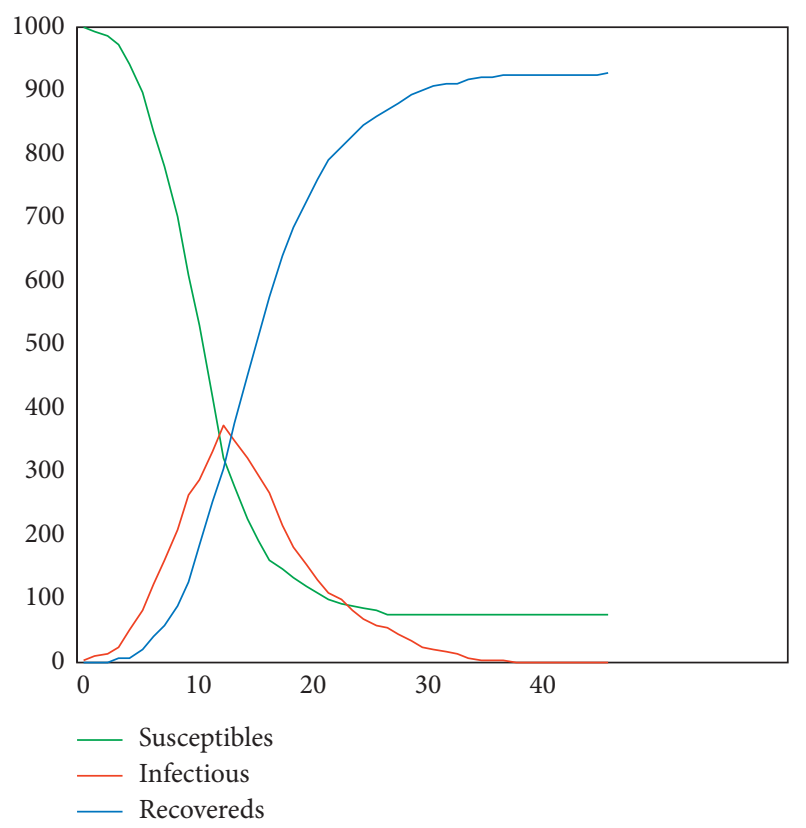

FIGURE 5: Government control measures and various social assistance strategies.

chain network substantially changed after the two root nodes, government control, and social assistance strategies had been implemented. The evaluation results of more accurate data were obtained by analyzing the model of the two root nodes, government control, and social assistance for clarification.

Figure 6 shows the interruption risk transmission process of the fresh cold chain network based on the SIR model. From the transmission process, it can be found that most of the companies in the network lack the ability to resist this risk; therefore, they are susceptible. Affected by interruption risk transmission, companies susceptible to infection risk become infectious. Some companies have taken effective measures to turn them from infectious companies to immunized companies. As time goes by, infected companies will absorb experience from the immunized companies and respond quickly to take effective and feasible measures, and eventually, immunized companies will grow faster. This is consistent with the spread of interruption risk in the network.

4.3. Sensitivity Analysis. It is necessary to adopt advanced measures to reduce the risk of the entire network and to accurately identify the key nodes of the revised fresh cold chain network interruption risk. Sensitivity analysis was used to investigate changes in the probability of intermediate nodes, subnodes, and root nodes in the fresh cold chain network interruption risk network, to show that the network is essential in each level node. The results are shown in Figure 7.

Different colors represent registration sensitivity. Red represents the highest agility, while blue represents the lowest sensitivity. Sensitivity analysis of interruption risk (Figure 7) showed that external risks, internal risks, and network collaboration risks are at a higher level of the network and are sensitive. Changes in the three risk nodes significantly impacted the network interruption risk in the revised fresh cold chain network. The sensitivities of fresh cold chain network interruption risk, external risk, internal risk, and network collaboration risk were 412, 206, 436, and 413 , respectively. Therefore, social assistance strategies and government control measures substantially influence fresh cold chain network interruption risk (internal risks). The sensitivity of risk nodes at different levels significantly varies due to the hierarchical network structure in the revised fresh cold chain network. Node sensitivities at the same level were analyzed and divided into nodes (sensitivity of the nodes at this level was substantial with logarithmic data processing. Root node sensitivity analysis is shown in Figures 8 and 9 .

In Figure 8, social risk, management risk, natural disasters, market risk, political risk, financial risk, credit risk, information risk, logistics risk, and technical risk were subnode sensitivities in descending order. In Figure 9, misinformation, price fluctuations, malicious arrears in finance, immorality, unqualified transportation, unmatched information, long product retention time, unfit storage technology, demand fluctuations, hidden information storage or transmission dangers, insufficient solvency, low production technology, product quality and safety, inventory realization risks, account receivable realization risks, investment and financing risks, inventory management risks, trade wars, insufficient employee responsibility, low level of information management, insufficient personnel reserves, environmental pollution, changes in policies and regulations, sudden accidents, social conflicts, natural disasters, and epidemics were root node sensitivities in a descending order. 


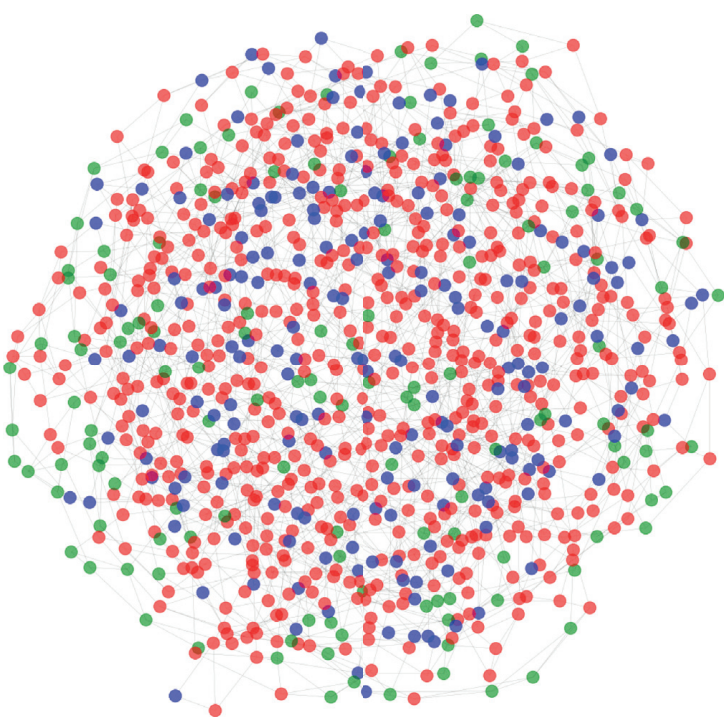

(a)

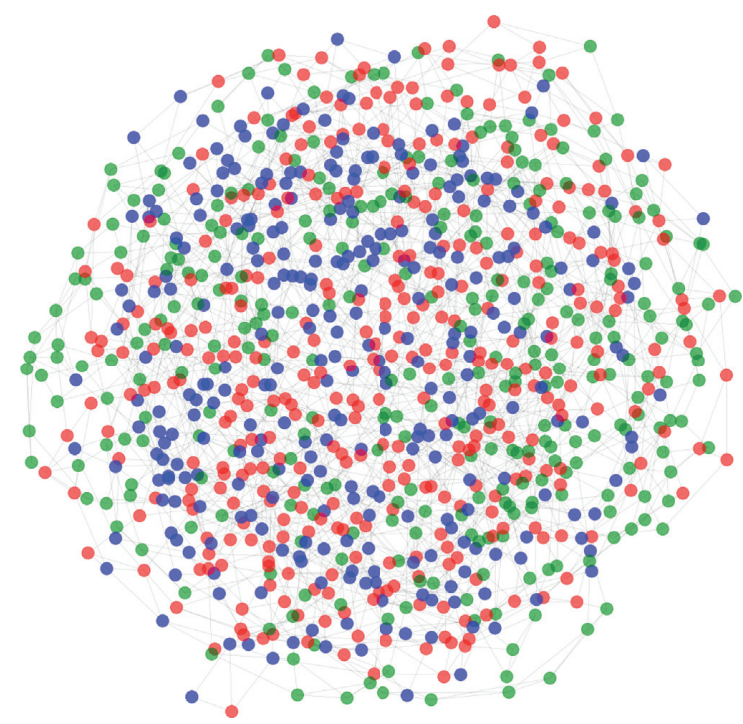

(b)

FIGURE 6: Interruption risk transmission process of fresh cold chain network based on the SIR model. The image on the left is when time $=8$, and the image on the right is the operation diagram when time $=12$. The green dot represents susceptibility, the red dot represents infectivity, and the blue dot represents recovery.

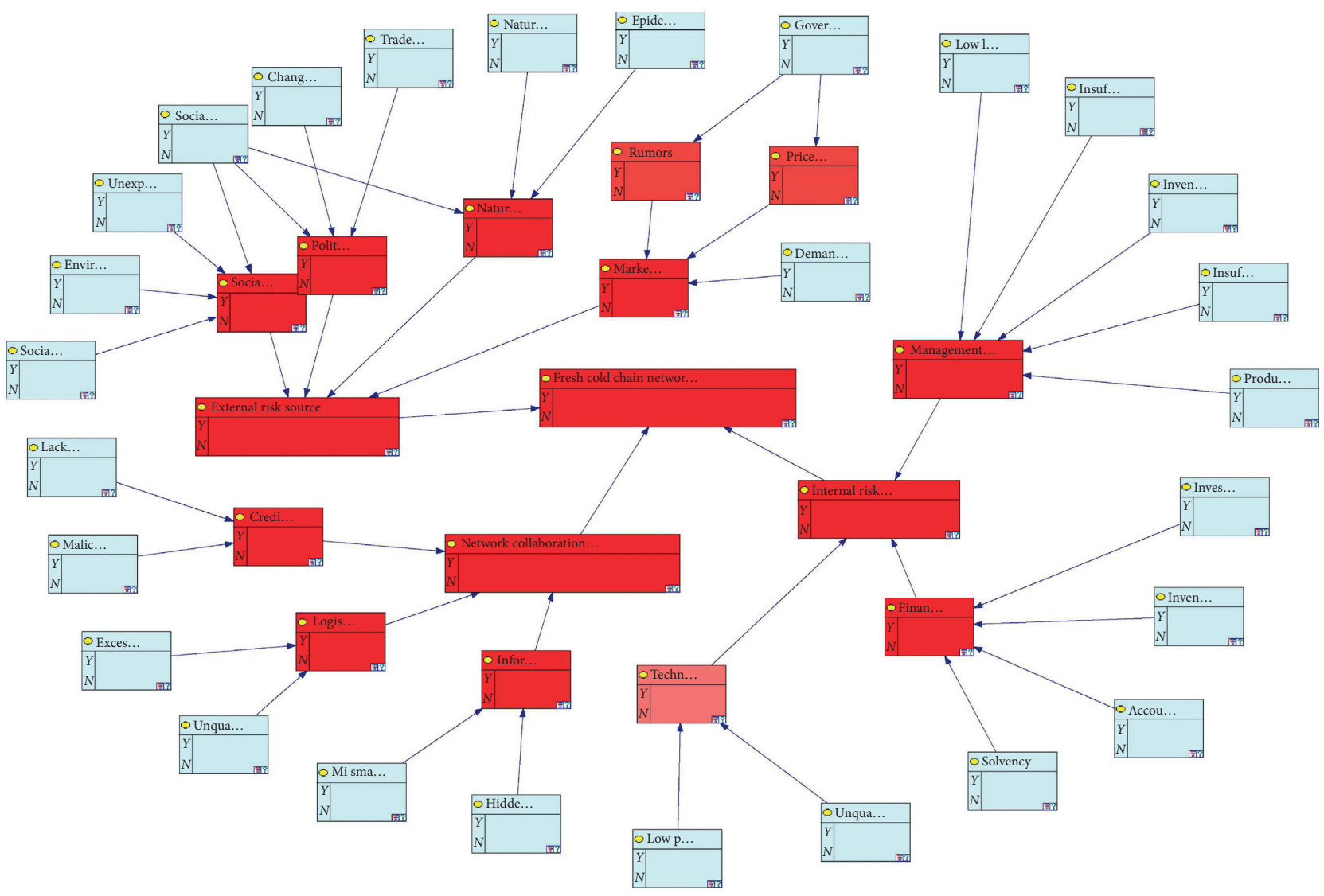

FiguRE 7: Revised fresh cold chain network interruption risk sensitivity analysis.

The government enforces necessary measures, such as macrocontrol, to control market stability, abnormal price fluctuations, and misinformation, thereby reducing the risks to 0.18 , with low occurrence possibilities. However, this study ignored the root node after misinformation and price fluctuations. The analysis is shown in Figure 10. 


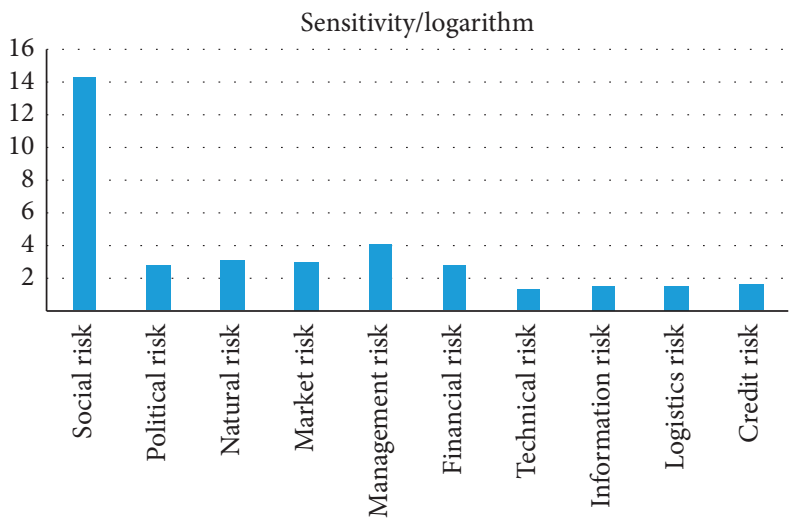

FIgURE 8: Sensitivity comparison diagram of subnodes.

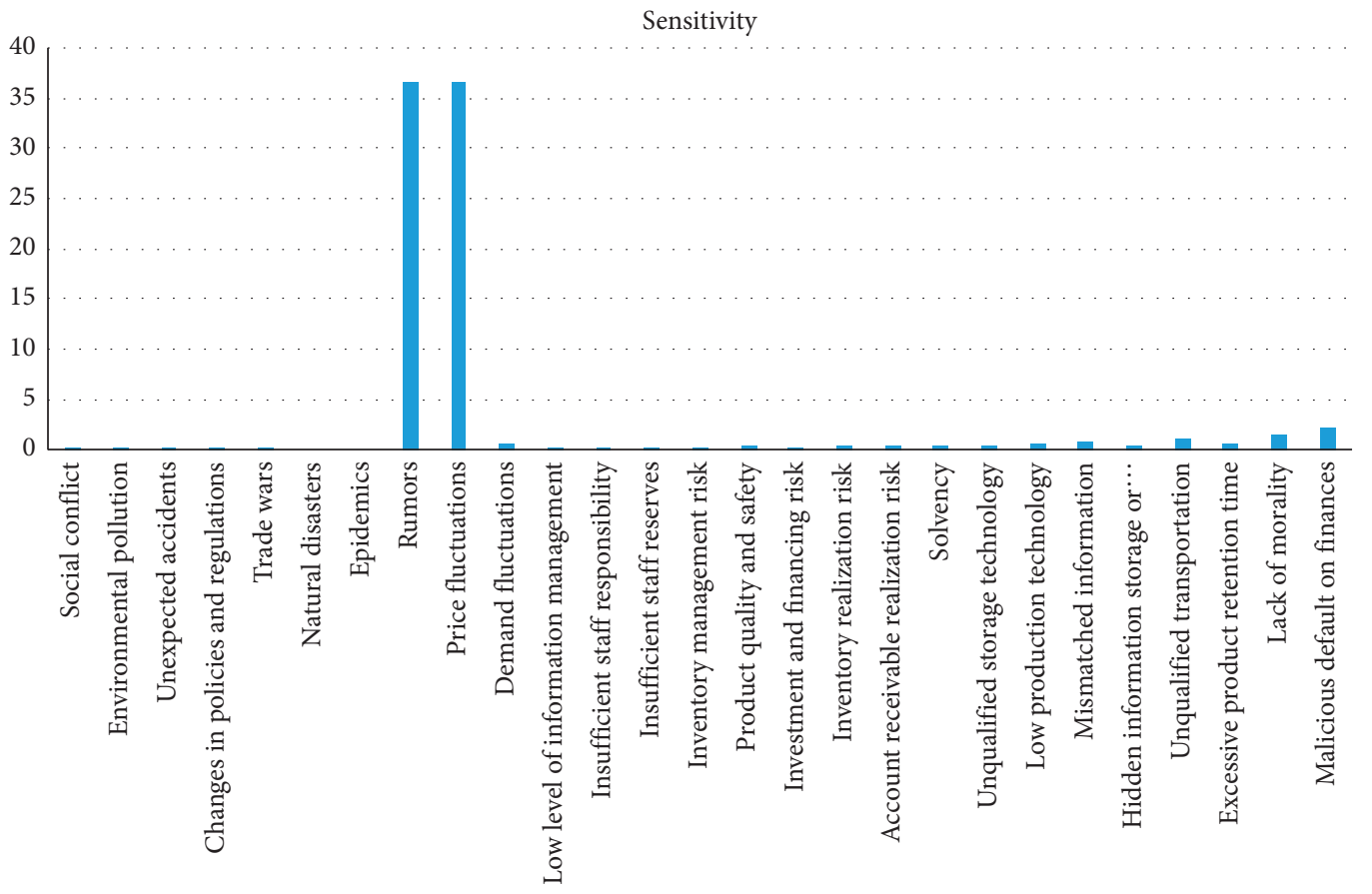

FIGURE 9: Sensitivity comparison diagram of evidence points.

The risk nodes, such as malicious default in finance, immorality, unqualified transportation, unmatched information, excessive product retention time, unfit storage technology, demand fluctuations, and hidden dangers of information storage or transmission, were found to be highly sensitive (Figure 10).

4.4. Suggestions. China's fresh cold chain network can be managed as follows:

Improving the Integrity of Supply Chain Network Members

By (i) relevant government agencies should strengthen information supervision, regulate information circulation channels, and strengthen circulating information and (ii) improving the credibility of each node enterprise and strengthening its ability to discern the market. Enterprises at each agricultural product supply chain node should enhance their technological level to meet Internet needs of the environment. The ability to distinguish between upstream and downstream companies and to accurately select reliable partners should also be improved [20].

Improving Distribution Efficiency and Quality of Logistics

A complete transportation and distribution monitoring system should be established to track and timely discover various fresh commodity problems during storage and distribution and reduce the possibility of deterioration and damage of fresh commodities caused by manual negligence or improper operation. 


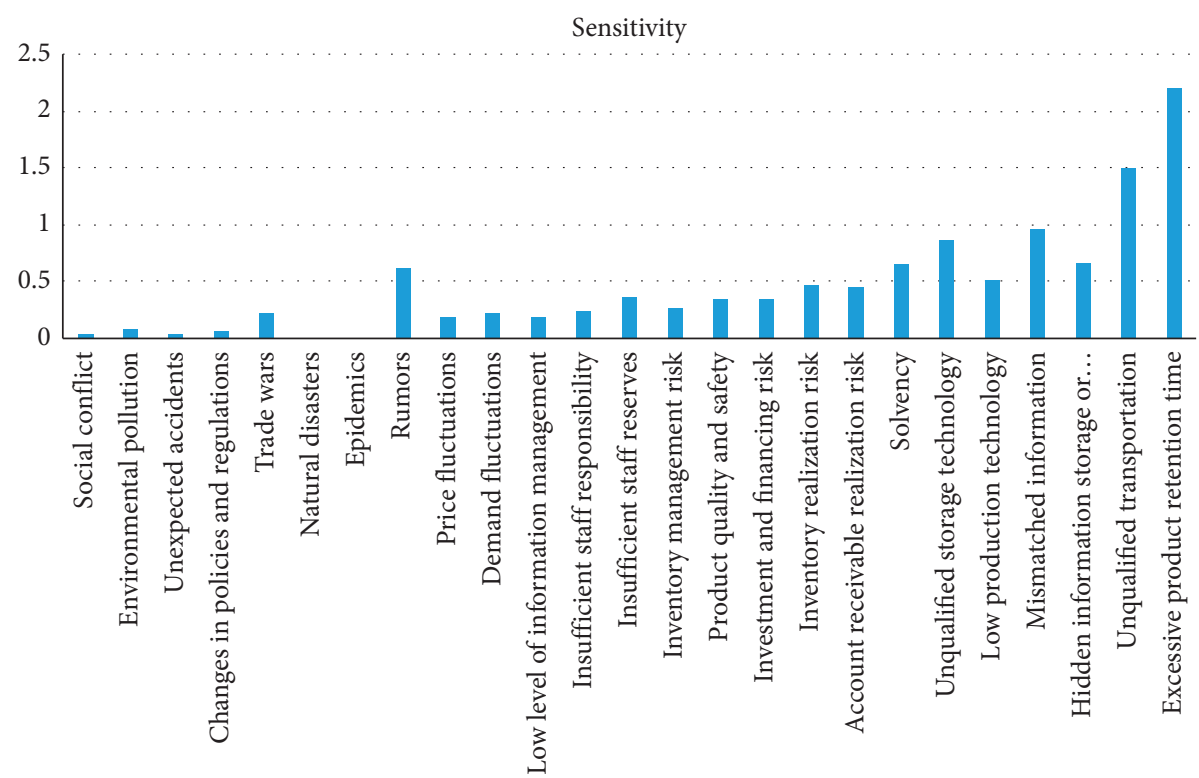

FIGURE 10: Sensitivity comparison of evidence points after controlling misinformation and price fluctuations.

Improving Cold Chain Storage Technology and the Quality of Cold Chain Technology

The storage process is the first link in the cold chain of fresh e-commerce products, and it should not directly affect sales operations. Many fresh products have strict storage conditions, especially in terms of temperature, humidity, and environment. Therefore, it is important to understand the different storage conditions for various fresh products with different physical and chemical properties and choose appropriate cold chain logistic equipment. Temperature and humidity requirements for fresh products should be noted during storage, and microorganisms should be regularly assessed to prevent deterioration.

Adopting Information Management Technology

Currently, computer informatization plays a critical role in information management. Computers are fast and accurate, simplify work, and save time, as well as material costs, and are, therefore, efficient and convenient in enterprise management. High-level information management reduces the loss of fresh agricultural products. Improving the connection between information technology and partner technology platforms is a modern method for realizing the smooth flow of cold chain logistics.

\section{Conclusions}

We used the Bayesian network for risk assessment of network interruption in the fresh cold chain network. We found the following:

(1) A Bayesian risk assessment model was established to evaluate the probability of network interruption risk affecting the fresh cold chain. In the root node, information management level was low, employee's sense of responsibility was insufficient, personnel reserves were insufficient, production technology was low, and the occurrence probability of unqualified storage technology was relatively high. In the subnodes, internal risks were found to significantly impact interruption risks mainly due to the relatively low development standards of Chinese enterprises.

(2) An SIR model was constructed to simulate interruption risk propagation in the fresh cold chain network. The roles of government control measures and various social assistance strategies were analyzed to determine the model that best suits China's national conditions.

(3) Sensitivity analysis was performed on probability assessment results of the risk of network interruption of the fresh cold chain network. The internal risk was the most sensitive. In the root node, the sensitivity of malicious delinquency financial risk was highest, implying that a small change in this factor can substantially influence credit risk, affecting network collaboration risk, and thereby fresh cold chain network interruption.

We used a Bayesian network prior probability model to determine the probability of risk occurrence, considered the dynamic nature of the network, supplemented by an SIR model to study risk transmission, and more accurately, assessed the source of disruption risk. This method assesses disruption risk from a new perspective, but its results are based on expert judgment, and model probability would be more accurately assessed by a posteriori probability model if it is verified with specific data.

\section{Data Availability}

The data used to support the findings of this study are available from the corresponding author upon request. 


\section{Conflicts of Interest}

The authors declare that they have no conflicts of interest.

\section{Acknowledgments}

This research was supported by the National Natural Science Foundation of China (51879211).

\section{References}

[1] B. Yan, P. Shi, and D. Ding, "Risk assessment and control of agricultural supply chain on internet of things," Journal of Industrial Engineering and Engineering Management, vol. 3, no. 12, pp. 196-202, 2014.

[2] F. Aqlan and S. S. Lam, "A fuzzy-based integrated framework for supply chain risk assessment," International Journal of Production Economics, vol. 161, no. 161, pp. 54-63, 2015.

[3] A. Díaz-Curbelo, Á. M. Gento, A. Redondo, and F. Aqlan, "A fuzzy-based holistic approach for supply chain risk assessment and aggregation considering risk interdependencies," Applied Sciences, vol. 9, no. 24, 2019.

[4] H. Zhang and M. Wang, "Failure risk identification model and simulation of supply chain under $\mathrm{O} 2 \mathrm{O}$ e-commerce model," Journal of System Simulation, vol. 11, no. 28, pp. 2747-2755, 2016.

[5] H. Yang and G. Hu, "Risk assessment of agricultural product supply chain based on fault tree and Bayesian network," Jiangsu Agricultural Sciences, vol. 5, no. 48, pp. 304-310, 2020.

[6] C. Zhao and K. Lang, "Fresh food logistics risk assessment based on Bayesian network," Systems Science and Mathematics Science, vol. 11, no. 40, pp. 2108-2124, 2018.

[7] J. Amundson, W. Faulkner, S. Sukumara, J. Seay, and F. Badurdeen, "A Bayesian network based approach for risk modeling to aid in development of sustainable biomass supply chains," 22nd European Symposium on Computer Aided Process Engineering, Elsevier, Amsterdam, Netherlands, pp. 152-156, 2012.

[8] J. Zhang and Z. Xu, "Research and development project risk probability assessment-based on dynamic Bayesian network," Soft Science, vol. 2, no. 31, pp. 131-135, 2015.

[9] C. Zheng, B. Peng, and W. Guo, "Operational risk modeling for cold chain logistics system: a Bayesian network approach," Kybernetes, vol. 50, no. 2, 2020.

[10] J. Dong, S. Wan, and S.-M. Chen, "Fuzzy best-worst method based on triangular fuzzy numbers for multi-criteria decisionmaking," Information Sciences, vol. 547, no. 547, pp. 1080-1104, 2021.

[11] S. Wan, X. Cheng, C. Chen, and J. Dong, " $L-R$ geometric consistency definition of triangular multiplicative preference relation in group decision making," Fuzzy Sets and Systems, vol. 409, pp. 85-113, 2021.

[12] W. Chen, W. Fan, X. Zhu, Q. Xie, and X. Yin, "DBN method for risk assessment of dairy products cold chain logistics," Journal of System Simulation, vol. 5, no. 31, pp. 936-945, 2019.

[13] W. Chen and M. Wang, "Dynamic risk assessment of pharmaceutical cold chain logistics in the internet of things environment," International Journal of Science Technology Management \& Research, vol. 1, pp. 215-220, 2020.

[14] S. Guohua, "Research on the fresh agricultural product supply chain coordination with supply disruptions," Discrete Dynamics in Nature and Society, vol. 2013, no. 5, 9 pages, Article ID 416790, 2013.
[15] J. Yang and H. Liu, "Research of vulnerability for fresh agricultural-food supply chain based on bayesian network," Mathematical Problems in Engineering, vol. 2018, Article ID 6874013, 17 pages, 2018.

[16] S.-P. Wan, W.-B. Huang Cheng, and J.-Y. Dong, "Interactive multi-criteria group decision-making with probabilistic linguistic information for emergency assistance of COVID-19," Applied Soft Computing Journal, vol. 107, Article ID 107383, 2021.

[17] A. Bradley, S. Sami, N. G. Hwei et al., "A predictive Bayesian network that risk stratifies patients undergoing Barrett's surveillance for personalized risk of developing malignancy," PLoS One, vol. 15, no. 10, 2020.

[18] C. D. Wickens, Engineering Psychology and Human Performance, Harper Collins Publishers Inc., New York, NY, USA, 1992.

[19] X. Zhang, Q. Zhang, J. Yang, Z. Cong, J. Luo, and H. Chen, "Safety risk analysis of unmanned ships in inland rivers based on a fuzzy Bayesian network," Journal of Advanced Transportation, vol. 2019, Article ID 4057195, 15 pages, 2019.

[20] L. Xuemei, "Study on the agricultural products supply chain risk based on internet of things environment," in Proceedings of the 2015 8th International Conference on Intelligent Computation Technology and Automation (ICICTA), Nanchang, China, June 2015. 